UDC 533.9.01

\title{
Generalized pair potential between charged particles in densesemiclassical plasma
}

\author{
Ramazanov T.S., Dzhumagulova K.N., Moldabekov Zh.A. \\ Al-FarabiKazakh National University, IETP, al-Farabi71,050040 Almaty, Kazakhstan
}

\begin{abstract}
The pair interaction potential of charged particles in a dense two-component plasmas, which takes into account quantum-mechanical effects, was obtained on the basis of the comparison of the quantum-mechanical Slater sum with the classical Boltzmann factor. The equations issued from this comparison were solved numerically and the spline approximation of these numerical results gives the interpolation formula for the pair interaction potential. The potential is valid in the temperature region of $10^{4}<\mathrm{K}<\mathrm{T}<10^{8} \mathrm{~K}$ and density region of $10^{21} \mathrm{~cm}^{-3}<n \leq 10^{24} \mathrm{~cm}^{-3}$. It was shown that at high temperatures and densities the results have a good agreement with well known models of Deutsch and Kelbg.
\end{abstract}

Keywords: semiclassical plasma, Slater sum, semiclassical interaction potential, dense two-component plasma. PACS number(s): 52.25.Mq, 52. 25. Ub

\section{Introduction}

The importance of the plasma state studying is caused by the fundamental interest in understanding of nature and importance of practical use. It is well known that the source of energy in stars is the thermonuclear reactions. An idea of the controlled thermonuclear fusion has led to the intensive research in the field of plasma physics. Present time the laboratory plasmas are investigated in a wide region of temperatures and pressures, from low-temperature gasdischarge plasma up to the dense plasma obtained by the laser beam [1], [2]. Some of the important reasons of a great interest to the investigation of the dense plasma properties are discussed in works [3-5].

According to the Big Bang theory all chemical elements were formed from the hydrogen atoms at the state of the high temperature plasma. Expanding plasma cooled and formed stars and planets. The hydrogen plasma consists of protons and electrons interacting by the Coulomb's law. Due to the quantum effects a deviation from the classical Coulomb's law for two point-like charges is observed at small distances. This deviation is caused by the Heisenberg uncertainty principle and the Pauli exclusion principle for fermions. Ebeling and coworkers reached significant progress on this problem [6-10]. High-temperature degenerate dense plasma was studied in detail by Deutsch. He suggested the pseudopotential which correctly takes into account the quantum effects of diffraction at high densities and temperatures $\mathrm{T}>10^{6} \mathrm{~K}[11-14]$.

\footnotetext{
* Corresponding author: e-mail: zhandos@physics.kz
}

In work [15] a pair interaction potential for the system of two particles was obtained by taking into account the quantum effects in the region from high temperatures $10^{8} \mathrm{~K}$ to low temperatures of a few tens of Kelvin in terms of the generalization of the Kelbg potential. A method of calculation of semiclassical potential which takes into account the effect of density is given in work [16].

In papers [17-20] the quantum effects, influencing on the interacting potential, were considered.

In works $[21,22]$ the dynamic and static properties of plasma were investigated with taking into account quantum effects.

It is known that quantum-mechanical analogue of the classical Boltzmann factor, which consists of the pair interaction potential, is the Slater sum. For $N$ particle system:

$$
S\left(r_{1}, \ldots, r_{N}\right)=c \sum_{n} \Psi_{n}^{*} e^{-\beta E_{n}} \Psi_{n},
$$

where:

$$
\begin{aligned}
c=\prod_{v} N_{v} ! \lambda_{v}^{3 N_{v}}, & \lambda_{v}^{2}=4 \pi \alpha_{v} \beta, \quad \alpha_{v}=\hbar^{2} / 2 m_{v}, \\
\beta & =1 / k_{B} T,
\end{aligned}
$$

$N_{v}$ is a number of particles of the $v$-th sort, which have a mass $m_{v}$ and thermal wavelength $\lambda_{v}$. The wave function is a properly symmetrized eigenfunction for the entire macroscopic system with eigenvalue $E_{n}$, where $n$ represents a complete set of quantum numbers. From equality of the Boltzmann factor and Slater sum one can obtain the semiclassical interaction po- 
tential. In Part 2 the equations issued from this comparison are presented. The results of their numerical solution and spline approximation are given in Part 3. The interpolation formula for the pair interaction potential are presented there.

The first step in calculation of the Slater sum is a choice of the wave function. For the determination of the wave function the TF approximation was used at the considered temperatures.

The equations for the Fourier transforms of the semiclassical potentials $\tilde{u}_{a b}(k)$ of two component plasma have the form:

$$
\begin{gathered}
\frac{\partial \tilde{u}_{i i}}{\partial \beta}=\tilde{v}-\delta \varepsilon \tilde{u}_{i i}-\tilde{Q}_{i i}-\frac{1}{2} \delta \rho \varepsilon \tilde{u}_{i i}^{2}-\frac{1}{2} \rho \varepsilon \tilde{u}_{i e}^{2}+\tilde{y}_{i i} \\
\frac{\partial \tilde{u}_{i e}}{\partial \beta}=-\tilde{v}-\frac{1}{2}(1+\delta) \varepsilon \tilde{u}_{i e}-\tilde{Q}_{i e}- \\
-\frac{1}{2} \delta \rho \varepsilon \tilde{u}_{i e} \tilde{u}_{i i}-\frac{1}{2} \rho \varepsilon \tilde{u}_{i e}\left(\tilde{u}_{e e}+1 / 2 \tilde{s}\right)+\tilde{y}_{i e} \\
\frac{\partial \tilde{u}_{e e}}{\partial \beta}=\tilde{v}-\frac{1}{2} \varepsilon \tilde{u}_{e e}-\tilde{Q}_{e e}-\frac{1}{2} \delta \rho \varepsilon \tilde{u}_{i e}^{2} \\
-\frac{1}{2} \rho \varepsilon \tilde{u}_{e e}\left(\tilde{u}_{e e}+\tilde{s}\right)+\tilde{y}_{e e}
\end{gathered}
$$

where: $\delta=m_{e} / m_{i}, \varepsilon=\varepsilon_{e}=\varepsilon_{i} / \delta, \varepsilon_{a}=\alpha_{a} x^{2}$, $Q_{a b}(r)=\frac{1}{4}\left(\alpha_{a}+\alpha_{b}\right) \nabla u_{a b}(r) \nabla u_{a b}(r)$

The $y_{a b}$ was obtained by the following formula:

$$
y_{a b}=X / S^{(2)}-3 / 2 \beta-1 / 4 \nabla u_{a b} \nabla u_{a b},
$$

where for two-particle system

$$
\begin{gathered}
S^{(2)}=c \sum_{n} \psi_{n}^{*} e^{-\beta E_{n}} \psi_{n}, \\
X=c \sum_{n} e^{-\beta E_{n}} \nabla \psi_{n}^{*} \nabla \psi_{n} .
\end{gathered}
$$

It should be noted that in this method the influence of the surrounding plasma on the diffraction effect between two particles is taken into account.

\section{Semiclassical potential}

The equations (3-5) were solved numerically using the boundary conditions:

$$
\tilde{u}_{a b} \stackrel{\beta \rightarrow 0}{\longrightarrow} 4 \pi \beta z_{a} z_{b} e^{2} / x^{2}
$$

Calculations were performed at temperatures $T \geq 10^{4} \mathrm{~K}$ and densities $n \geq 10^{21} \mathrm{~cm}^{-3}$. Using these numerical values of the interaction potentials for the considered range of temperatures and densities following interpolation formula was obtained.

$$
\begin{gathered}
u_{a b}(r)=\frac{z_{a} z_{b} e^{2}}{r} \times \\
\times\left\{1-\tanh \left(\sqrt{2} \frac{\lambda_{a b}^{2}}{a^{2}+b r^{2}}\right) e^{-\tanh \left(\sqrt{2} a_{a b}^{2}\right)\left(a^{2}+b r^{2}\right)}\right\}\left(1-e^{-r / \lambda_{a b}}\right)
\end{gathered}
$$

where $\lambda_{a b}=\hbar / \sqrt{4 \pi m_{a b} k_{B} T}$ thermal wave length, $m_{a b}=$ $=m_{a} m_{b} /\left(m_{a}+m_{b}\right), a=(3 / 4 \pi n)^{1 / 3}$ is an average interparticle distance and $b=0.033$.

In the $\operatorname{limit} T \rightarrow \infty$ potential (10) coincides with the Deutsch potential [11]:

$$
\left.u_{a b}\right|_{T \rightarrow \infty}=\frac{z_{a} z_{b}}{r}\left(1-\exp \left[-r / \lambda_{\alpha \beta}\right]\right)
$$

In figures 1-3 the semiclassical pair interaction potential (10) and numerical data obtained by solving the equations (3-5) for several temperatures are presented. One can see that at increase in temperature potential (10) tends to the Deutsch potential. In figures 4-7, where $\Gamma=\beta e^{2} / a$, it is shown that the many particles effect on the diffraction term is significant in the dense plasma.

The potential (10) can be used for plasma at low temperature $T<10^{4} \mathrm{~K}$ if density is enough high and plasma is fully ionized due to the Mott transition. For electron-electron pair the difference in magnitude between potentials (11) and (10) when $r \rightarrow 0$ for the different values of density and temperature is shown in the figure 8 . In the figure 9 the same difference in magnitude when $r \rightarrow 0$ for the different values of the density parameter and the coupling parameter is shown. It is seen that the difference in magnitude is larger for the dense nonideal plasmas. The values of the potential (10) at short distance $(r \rightarrow 0)$ for the different values of density and temperature are shown in the figure 10.

Tables 1-5 show the comparison of the obtained results with the data presented in the work of Barker [24]. These tables show that the density-effects play a significant role when $r_{s}<3$. With increase in density parameter the pseudopotential (10) approaches to the effective potential of Barker, which takes into account the effect of diffraction for two isolated charged particles. 


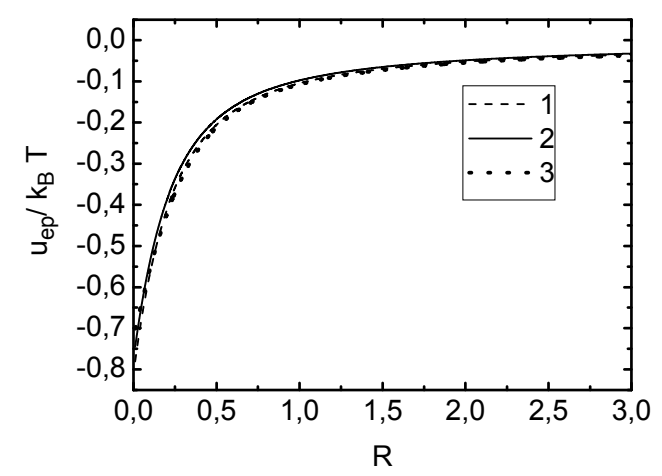

Figure 1 - Proton-electron pair interaction potential. Curve 1 is the Deutschpotential (11), curve 2 is the semiclassical potential (10), 3 is thenumerical data. $R=r / a_{0}, T=3.16 \cdot 10^{6} \mathrm{~K}$.

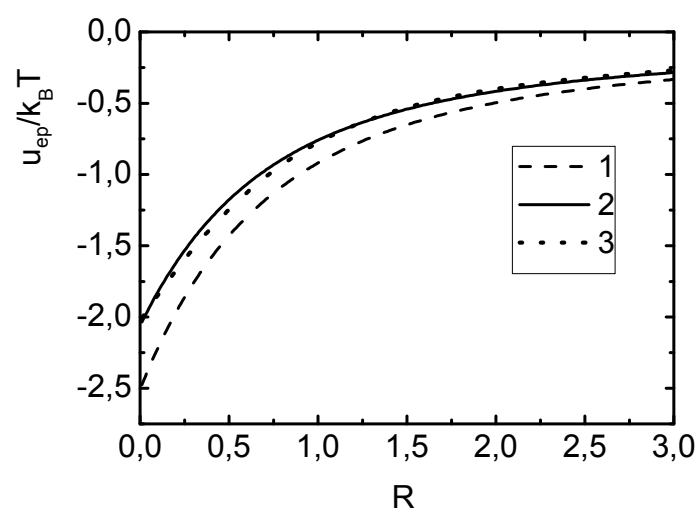

Figure 2 - Proton-electron pair interaction potential. Curve 1 is the Deutsch potential (11), curve 2 is the semiclassical potential (10), 3 is the numerical data. Where $R=r / a_{0}, T=3.5 \cdot 10^{5} \mathrm{~K}$

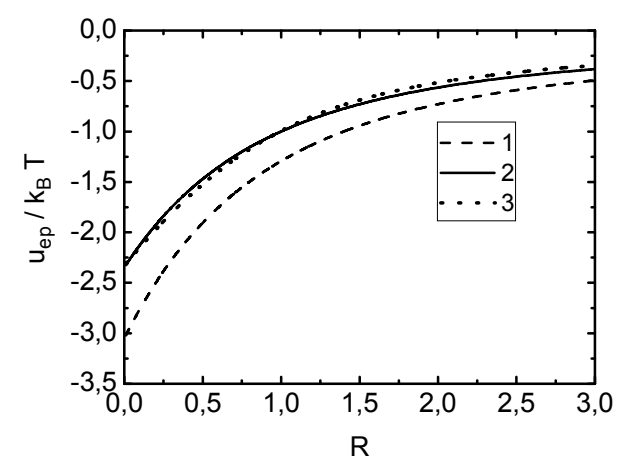

Figure 3 - Proton-electron pair interaction potential. Curve 1 is the Deutsch potential (11), curve 2 is the semiclassical potential (10), 3 is the numerical data.

Where $R=r / a_{0}, T=2.1 \cdot 10^{5} \mathrm{~K}$

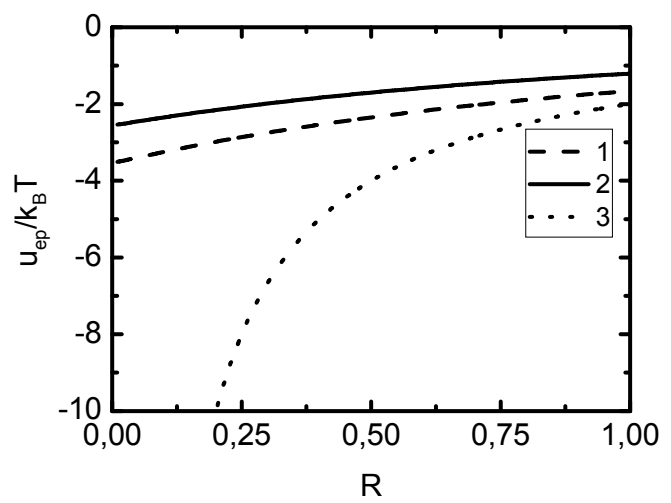

Figure 4 - Proton-electron pair interaction potential. Curve 1 is the Deutsch potential (11), curve 2 is the semiclassical potential (10),3 is the Coulomb potential. Where $R=r / a_{0}, \Gamma=$ $2, r_{s}=1$.

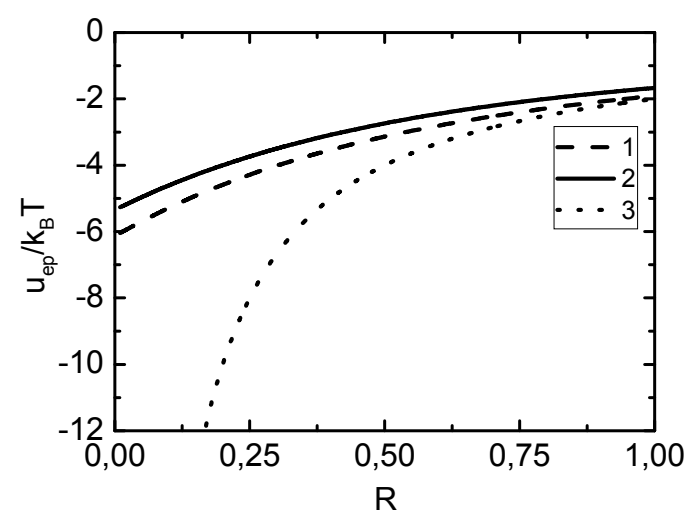

Figure 5 - Proton-electron pair interaction potential. Curve 1 is the Deutsch potential (11), curve 2 is the semiclassical potential (10), 3 is the Coulomb potential. Where $R=r / a_{0}, \Gamma=$ $2, r_{s}=3$.

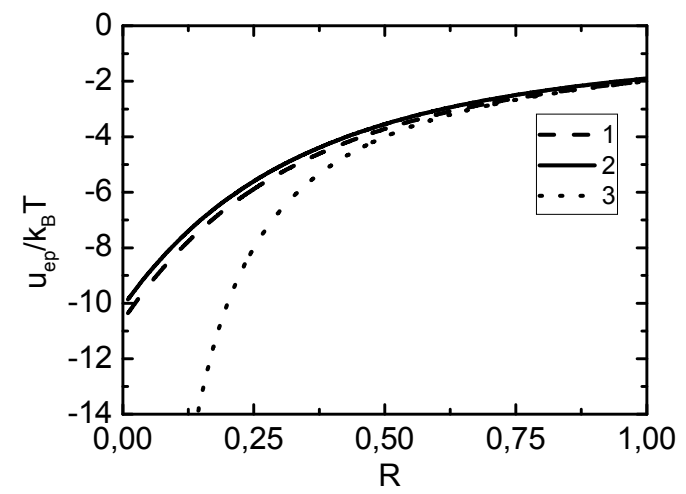

Figure 6 - Proton-electron pair interaction potential. Curve 1 is the Deutsch potential (11), curve 2 is the semiclassical potential (10), 3 is the Coulomb potential. Where $R=r / a_{0}, \Gamma=$ $2, r_{s}=9$. 


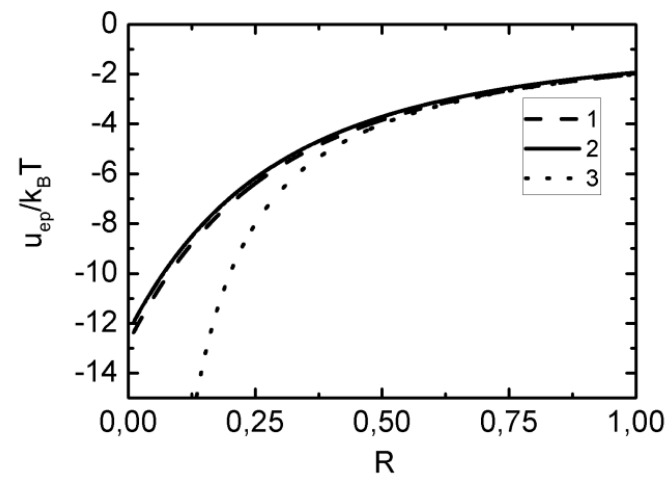

Figure 7 - Proton-electron pair interaction potential.

Curve 1 is the Deutsch potential (11), curve 2 is the semiclassical potential (10), 3 is the Coulomb potential. Where $R=r / a_{0}, \Gamma=2, r_{s}=13$.

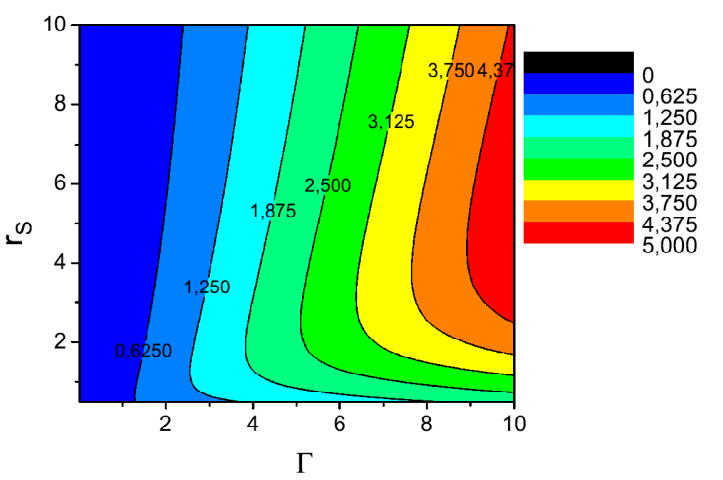

Figure $9-$ The $r_{s}=\Gamma$ profile of the difference in magnitudes (in the unit of $k_{B} T$ ) of potentials (11) and (10) when $r \rightarrow 0$.

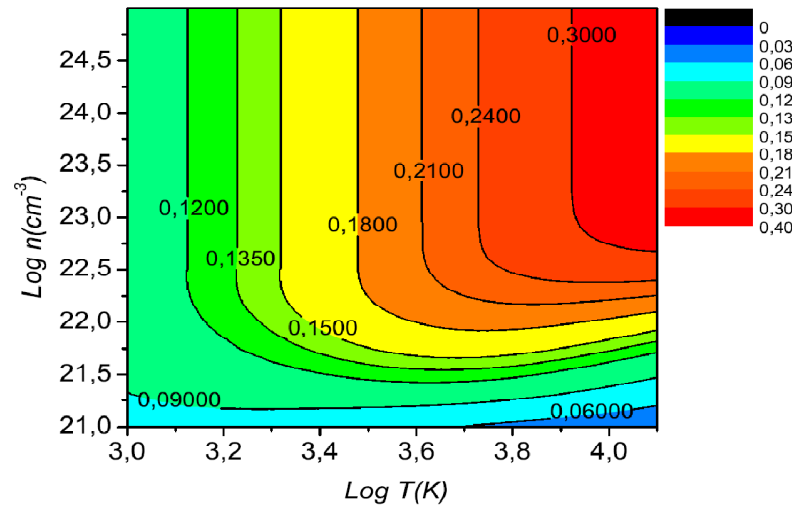

Figure 8 - The $n$-T profile of the difference in magnitudes (in the unit of Rydberg) of potentials (11) and (10) when $r \rightarrow 0$

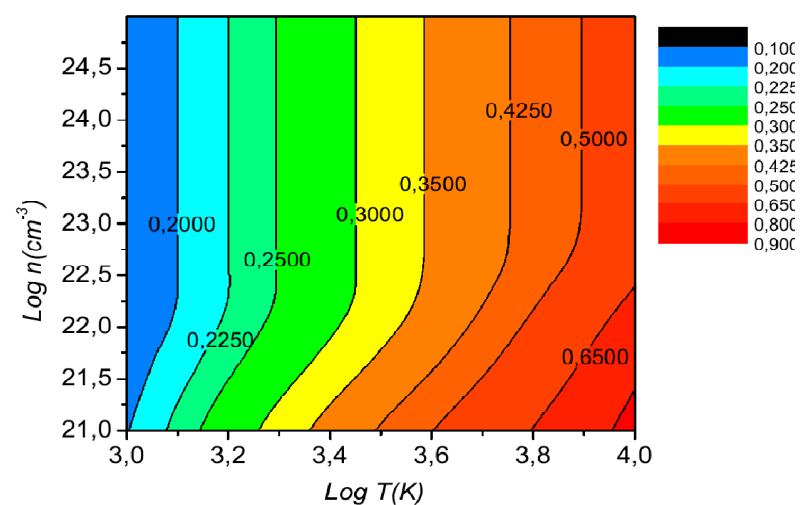

Figure $10-$ The n-T profile of the magnitude of the potential (10) (in the unit of Rydberg) when $r \rightarrow 0$.

Table 1 - The effective interaction potential of the proton-electron in unit of $\beta=1 / k_{B} T$ at temperature $T=5 \times 10^{4} K$, for several values of $r_{S}$. Here $u_{e p}^{B a r \text { ker }}$ are results of Barker and $\Delta u_{e p}=u_{e p}-u_{e p}^{B a r \text { ker }}$.

\begin{tabular}{|c|c|c|c|c|c|c|c|}
\hline$r\left(a_{B}\right)$ & $u_{e p}^{r_{s}=1}$ & $u_{e p}^{r_{s}=3}$ & $u_{e p}^{r_{s}=10}$ & $u_{e p}^{\text {Bar } \mathrm{ker}}$ & $\Delta u_{e p}^{r_{s}=1}$ & $\Delta u_{e p}^{r_{s}=3}$ & $\Delta u_{e p}^{r_{s}=10}$ \\
\hline 0.0 & -1.2868 & -1.7988 & -1.9777 & -2.3852 & 1.0984 & 0.5864 & 0.4075 \\
\hline 0.5 & -1.0132 & -1.4124 & -1.5572 & -2.0769 & 1.0637 & 0.6605 & 0.5167 \\
\hline 1.0 & -0.8143 & -1.1383 & -1.2515 & -1.7630 & 0.9487 & 0.6247 & 0.5115 \\
\hline 1.5 & -0.6674 & -0.9330 & -1.0258 & -1.4581 & 0.7907 & 0.5251 & 0.4323 \\
\hline 2.0 & -0.5573 & -0.7790 & -0.8565 & -1.1747 & 0.6174 & 0.3957 & 0.3182 \\
\hline 2.5 & -0.4734 & -0.6618 & -0.7276 & -0.9316 & 0.4582 & 0.2698 & 0.204 \\
\hline 3.0 & -0.4084 & -0.5710 & -0.6278 & -0.7442 & 0.3358 & 0.1732 & 0.1164 \\
\hline 3.5 & -0.3574 & -0.4995 & -0.5492 & -0.6119 & 0.2545 & 0.1123 & 0.0627 \\
\hline 4.0 & -0.3165 & -0.4425 & -0.4865 & -0.5208 & 0.2043 & 0.0783 & 0.0343 \\
\hline 4.5 & -0.2835 & -0.3962 & -0.4357 & -0.4558 & 0.1723 & 0.0596 & 0.0201 \\
\hline 5.0 & -0.2562 & -0.3582 & -0.3938 & -0.4067 & 0.1505 & 0.0485 & 0.0129 \\
\hline 5.5 & -0.2336 & -0.3265 & -0.3590 & -0.3679 & 0.1343 & 0.0414 & 0.0089 \\
\hline 6.0 & -0.2145 & -0.2998 & -0.3296 & -0.3359 & 0.1214 & 0.0063 & 0.0063 \\
\hline 6.5 & -0.1981 & -0.2770 & -0.3046 & -0.3098 & 0.1117 & 0.0328 & 0.0052 \\
\hline 7.0 & -0.1841 & -0.2574 & -0.2830 & -0.2871 & 0.103 & 0.0041 & 0.0041 \\
\hline 7.5 & -0.1720 & -0.2403 & -0.2642 & -0.2677 & 0.0957 & 0.0027 & 0.0035 \\
\hline
\end{tabular}


Table 2 - The effective interaction potential of the proton-electron in unit of $\beta=1 / k_{B} T$ at temperature $T=10^{5} K$, for several values of $r_{S}$. Here $u_{e p}^{B a r \text { ker }}$ are results of Barker and $\Delta u_{e p}=u_{e p}-u_{e p}^{B a r \text { ker }}$.

\begin{tabular}{|c|c|c|c|c|c|c|c|}
\hline$r\left(a_{B}\right)$ & $u_{e p}^{r_{s}=1}$ & $u_{e p}^{r_{S}=3}$ & $u_{e p}^{r_{s}=10}$ & $u_{e p}^{\text {Bar ker }}$ & $\Delta u_{e p}^{r_{s}=1}$ & $\Delta u_{e p}^{r_{s}=3}$ & $\Delta u_{e p}^{r_{s}=10}$ \\
\hline 0,0 & -2.0007 & -2.6760 & -2.8109 & -2.8109 & 0.8039 & 0.1349 & 0.0000 \\
\hline 0,5 & -1.4355 & -1.9200 & -2.0170 & -2.5566 & 1.1211 & 0.6366 & 0.5396 \\
\hline 1,0 & -1.0723 & -1.4348 & -1.5065 & -1.9573 & 0.885 & 0.5225 & 0.4508 \\
\hline 1,5 & -0.8316 & -1.1123 & -1.1683 & -1.4448 & 0.6132 & 0.3325 & 0.2765 \\
\hline 2,0 & -0.6670 & -0.8920 & -0.9370 & -1.0714 & 0.4044 & 0.1794 & 0.1344 \\
\hline 2,5 & -0.5507 & -0.7365 & -0.7736 & -0.8321 & 0.2814 & 0.0956 & 0.0585 \\
\hline 3,0 & -0.4660 & -0.6232 & -0.6546 & -0.6806 & 0.2146 & 0.0574 & 0.026 \\
\hline 3,5 & -0.4023 & -0.5381 & -0.5652 & -0.5782 & 0.1759 & 0.0411 & 0.013 \\
\hline 4,0 & -0.3533 & -0.4726 & -0.4964 & -0.5037 & 0.1504 & 0.0311 & 0.0073 \\
\hline 4,5 & -0.3146 & -0.4208 & -0.4420 & -0.4466 & 0.132 & 0.0258 & 0.0046 \\
\hline 5,0 & -0.2834 & -0.3791 & -0.3982 & -0.4012 & 0.1178 & 0.0221 & 0.003 \\
\hline
\end{tabular}

Table 3 - The effective interaction potential of the proton-electron in unit of $\beta=1 / k_{B} T$ at temperature $T=5 \times 10^{5} K$, for several values of $r_{S}$. Here $u_{e p}^{B a r \text { ker }}$ are results of Barker and $\Delta u_{e p}=u_{e p}-u_{e p}^{B a r \text { ker }}$.

\begin{tabular}{|c|c|c|c|c|c|c|c|}
\hline$r\left(a_{B}\right)$ & $u_{e p}^{r_{s}=1}$ & $u_{e p}^{r_{S}=3}$ & $u_{e p}^{r_{s}=10}$ & $u_{e p}^{\text {Bar ker }}$ & $\Delta u_{e p}^{r_{s}=1}$ & $\Delta u_{e p}^{r_{S}=3}$ & $\Delta u_{e p}^{r_{s}=10}$ \\
\hline 0,0 & -5.7445 & -6.2470 & -6.3107 & -6.3066 & 0.5621 & 0.0596 & 0.0041 \\
\hline 0,5 & -2.8900 & -3.1429 & -3.1748 & -3.7160 & 0.8260 & 0.5731 & 0.5412 \\
\hline 1,0 & -1.7435 & -1.8961 & -1.9153 & -2.0357 & 0.2922 & 0.1397 & 0.1204 \\
\hline 1,5 & -1.2034 & -1.3087 & -1.3220 & -1.3416 & 0.1382 & 0.0328 & 0.0196 \\
\hline 2,0 & -0.9100 & -0.9884 & -0.9985 & -1.0026 & 0.0926 & 0.0142 & 0.0041 \\
\hline 2,5 & -0.7300 & -0.7919 & -0.8000 & -0.8011 & 0.0711 & 0.0092 & 0.0011 \\
\hline
\end{tabular}

Table 4 - The effective interaction potential of the proton-electron in unit of $\beta=1 / k_{B} T$ at temperature $T=10^{6} K$, for several values of $r_{S}$. Here $u_{e p}^{B a r \text { ker }}$ are results of Barker and $\Delta u_{e p}=u_{e p}-u_{e p}^{B a r \text { ker }}$.

\begin{tabular}{|c|c|c|c|c|c|c|c|}
\hline$r\left(a_{B}\right)$ & $u_{e p}^{r_{S}=1}$ & $u_{e p}^{r_{S}=3}$ & $u_{e p}^{r_{S}=10}$ & $u_{e p}^{\text {Bar } \mathrm{ker}}$ & $\Delta u_{e p}^{r_{S}=1}$ & $\Delta u_{e p}^{r_{S}=3}$ & $\Delta u_{e p}^{r_{S}=10}$ \\
\hline 0,0 & -8.5069 & -8.8839 & -8.9290 & -8.9189 & 0.4120 & 0.0350 & 0.0101 \\
\hline 0,5 & -3.4041 & -3.5550 & -3.5730 & -3.9687 & 0.5646 & 0.4137 & 0.3957 \\
\hline 1,0 & -1.8850 & -1.9685 & -1.9785 & -2.0163 & 0.1313 & 0.0478 & 0.0378 \\
\hline 1,5 & -1.2698 & -1.3261 & -1.3328 & -1.3351 & 0.0653 & 0.0090 & 0.0023 \\
\hline 2,0 & -0.9534 & -0.9956 & -1.0007 & -1.0001 & 0.0467 & 0.0045 & 0.0006 \\
\hline
\end{tabular}


Table 5 - The effective interaction potential of the proton-electron in unit of $\beta=1 / k_{B} T$ at temperature $T=5 \times 10^{6} K$, for several values of $r_{S}$. Here $u_{e p}^{B a r \text { ker }}$ are results of Barker and $\Delta u_{e p}=u_{e p}-u_{e p}^{B a r k e r}$.

\begin{tabular}{|c|c|c|c|c|c|c|c|}
\hline$r\left(a_{B}\right)$ & $u_{e p}^{r_{s}=1}$ & $u_{e p}^{r_{s}=3}$ & $u_{e p}^{r_{s}=10}$ & $u_{e p}^{\text {Bar } \mathrm{ker}}$ & $\Delta u_{e p}^{r_{s}=1}$ & $\Delta u_{e p}^{r_{s}=3}$ & $\Delta u_{e p}^{r_{s}=10}$ \\
\hline 0,0 & -19.7771 & -19.9536 & -19.9739 & -19.9432 & 0.1661 & 0.0104 & 0.0307 \\
\hline 0,5 & -3.9385 & -3.9736 & -3.9776 & -4.0064 & 0.0391 & 0.0328 & 0.0288 \\
\hline 1,0 & -1.9828 & -2.0004 & -2.0024 & -1.9996 & 0.0163 & 0.0008 & 0.0028 \\
\hline 1,5 & -1.3218 & -1.3336 & -1.3350 & -1.3330 & 0.0112 & 0.0006 & 0.0020 \\
\hline
\end{tabular}

At high densities $n>10^{22} \mathrm{~cm}^{-3}$ the semiclassical potential (10) weakly depends on the change in the density (see fig.10). In this case the semiclassical potential (10) can be interpolated by the following formula:

$$
\begin{gathered}
u_{a b}(r)=\frac{z_{a} z_{b}}{r} \times \\
\times\left\{1-\tanh \left(\sqrt{2} \frac{\lambda_{a b}^{2}}{a_{B}^{2}+b r^{2}}\right) e^{-\tanh \left(\sqrt{2} \lambda_{a b}^{2} /\left(a_{B}^{2}+b r^{2}\right)\right)}\right\}\left(1-e^{-r / \lambda_{a b}}\right)
\end{gathered}
$$

For the sake of completeness it is desired to account the quantum mechanical symmetry effect arising from the interaction of the identical particles as well as the diffraction effect. Its influence on the interaction is weaker then the diffraction effect but can be quite large at high densities. The symmetric effective potential for the mixture of the ideal gases of independent electrons has the following form [15]:

$$
u_{e e}(r)=-k_{B} T \ln \left(1-\frac{1}{2} \exp \left(-\frac{r^{2}}{\lambda_{e e}^{2}}\right)\right) \text {. }
$$

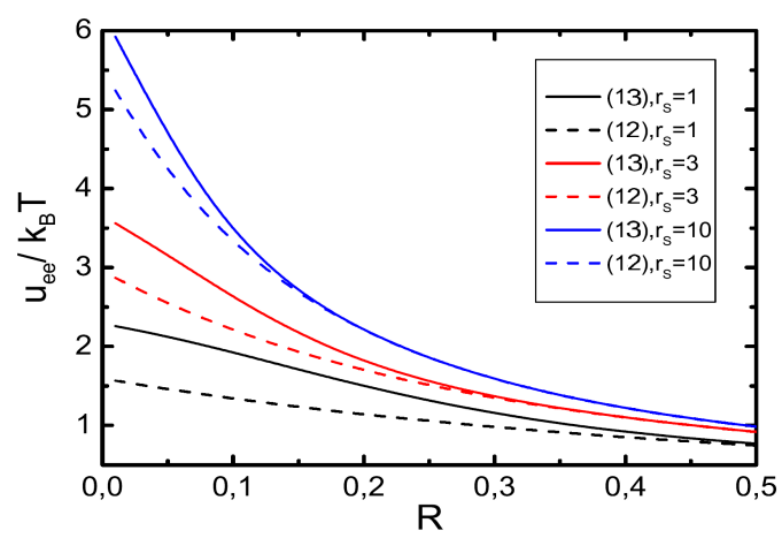

Figure 11 - Electron-electron pair interaction potentials. Here $R=r / a, \quad \Gamma=0.5$.

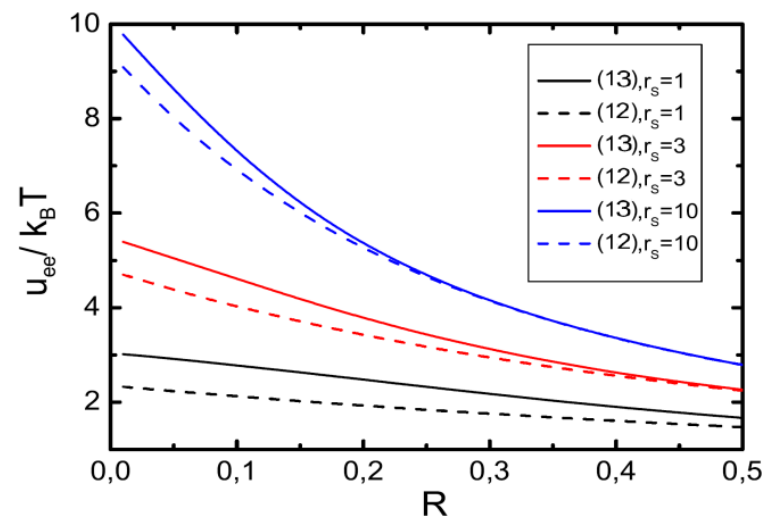

Figure 12 - Electron-electron pair interaction potentials. Here $R=r / a, \quad \Gamma=0.5$.

Hence, according to (12) the following formula has been obtained for the total pair interaction potential in the dense nonideal plasma:

$$
\begin{aligned}
& u_{a b}(r)=\frac{z_{a} z_{b}}{r} \times \\
& \times\left\{1-\tanh \left(\sqrt{2} \frac{\lambda_{a b}^{2}}{a_{B}^{2}+b r^{2}}\right) e^{-\tanh \left(\sqrt{2} \lambda_{a b}^{2} /\left(a_{B}^{2}+b r^{2}\right)\right)}\right\} \times \\
& \times\left(1-e^{-r / \lambda_{a b}}\right)-k_{B} T \ln \left(1-\frac{1}{2} \exp \left(-\frac{r^{2}}{\lambda_{e e}^{2}}\right)\right)
\end{aligned}
$$

It should be noted that the diffraction effect in the many particles system for pair interaction potential is included in the first term and the second term is responsible for symmetry effect between fermions in plasma. The comparison of the potentials (12) without symmetric term and (13) with symmetric term is shown in figures 11,12 . It is seen that the contribution of the symmetry effect diminishes with decrease in density and with increase in temperature. 


\section{Conclusions}

Generalized potential of pair interaction for the dense semiclassical plasma was obtained. It correctly takes into account diffraction effects in the many particles system in a wide region of temperatures and densities. Semiclassical potential has a good agreement with the Deutsch potential at high temperatures. This potential can be modified by the ad- dition of the term taking into account the symmetry effects. As the result the semiclassical pair interaction potential which takes into account both diffraction effect and symmetry effect for dense nonideal plasma was obtained.

This work was supported by the Ministry of Education and Science of the Republic of Kazakhstan under grant 1415/GF2 (IPC-21,2013).

\section{References}

[1] C. Deutsch, G. Maynard, M. Chabot et al.,The Open Plasma Physics Journal. 3, (2010).

[2] H. Nersisyan, C. Deutsch, Laser And Particle Beams. 29, (2011).

[3] W. Lorenzen , B. Holst , R. Redmer , Phys. Rev. B.82, 195107 (2010)

[4] B. Holst, M. French, R. Redmer, Phys. Rev. B .83, 235120 (2011)

[5] W. Lorenzen , B. Holst , R. Redmer , Phys. Rev. B .84, 235109 (2011)

[6] W. Ebeling, Ann. Phys. 19, 104(1967).

[7] W. Ebeling, G. Kelbg, and R. Sandig, Beitr. Plasma Phys. \textbf $\{10\}, 507(1970)$.

[8] V. S. Filinov, M. Bonitz, W. Ebeling, and V. E. Fortov, Plasma Phys. Controled Fusion. 43, 743(2001).

[9] S. A. Trigger, W. Ebeling, V. S. Filinov, V. E. Fortov, and M. Bonits, JETP. 96, 465(2003).

[10] W. Ebeling ,H. Hache , H. Juranek, R. Redmer and G. Ropke, Contri. Plasma Phys.45, 160(2005).

[11] C. Deutsch, Ann. Phys. (N.Y.)115, 404 (1971).

[12] C. Deutsch, Phys. Letters,60A, 4 (1977).

[13] N. Grandjouan, C. Deutsch, Phys. Rev. A11, 522(1974).
[14] H. Minoo, M.M. Gombert and C. Deutsch, Phys. Rev. A, 23, 2(1981)

[15] A.V. Filinov, V.O. Golubnychiy, M. Bonitz, W. Ebeling, J.W. Dufty, Phys. Rev E 70,046411 (2004).

[16] M.A. Pokrant, A.A. Broyles and T. Dunn, Phys. Rev. A10, 379 (1974).

[17] V. Golubnychiy, M. Bonitz, D. Kremp, M. Schlanges, Phys. Rev. E, 64, 016409 (2001)

[18] V.S. Filinov, P. Thomas, I. Varga, T. Meier, M. Bonitz, V.E. Fortov, S.W. Koch, Phys. Rev. B 65, 165124 (2002).

[19] T.S. Ramazanov, K.N. Dzhumagulova, Yu.A. Omarbakiyeva, Phys. Plasmas 9, 092702-1-4 (2005).

[20] T.S. Ramazanov, K.N. Dzhumagulova, Phys. Plasmas 9, 3758 (2002).

[21] T. Raitza ,G. Roepke, H. Reinholz et al. Phys. Rev. E. 84, 036406 (2011).

[22] M. Steinberg, W. Ebeling, J. Ortner, Phys. Rev. E. 61, 2290 (2000).

[23] Zh.A. Moldabekov., T.S. Ramazanov., K.N. Dzhumagulova, Contrib. Plasma Phys., 3, 207 - 210 (2012).

[24] A. A. Barker, J. Chem. Phys. 1751 (1971). 